\title{
Cerebrospinal fluid levels of amyloid-beta oligomers in patients with idiopathic normal pressure hydrocephalus are elevated before shunt surgery but decrease afterward
}

\author{
Kaito Kawamura ( $\nabla$ k-kawamu@juntendo.ac.jp ) \\ Juntendo University https://orcid.org/0000-0001-9956-5797 \\ Masakazu Miyajima \\ Juntendo University \\ Madoka Nakajima \\ Juntendo University \\ Mitsuyasu Kanai \\ Mihara Memorial Hospital \\ Yumiko Motoi
}

Juntendo University

Shuko Nojiri

Juntendo University

Chihiro Akiba

Juntendo University

Ikuko Ogino

Juntendo University

Hanbing Xu

Juntendo University

Chihiro Kamohara

Juntendo University

Shinya Yamada

Juntendo University

Kostadin Karagiozov

Juntendo University

Hajime Arai

Juntendo University

Research 
Keywords: idiopathic normal pressure hydrocephalus, amyloid-beta, amyloid-beta oligomer, cerebrospinal fluid

Posted Date: June 15th, 2020

DOI: https://doi.org/10.21203/rs.3.rs-35068/v1

License: (c) (1) This work is licensed under a Creative Commons Attribution 4.0 International License. Read Full License 


\section{Abstract \\ Background}

The amyloid-beta $(A B)$ oligomer has strong neurotoxicity and is associated with cognitive impairment in Alzheimer's disease (AD). However, its role in patients with idiopathic normal pressure hydrocephalus (iNPH) is poorly understood. We hypothesised that cerebrospinal fluid (CSF) stagnation leads to $A \beta$ oligomer accumulation in patients with iNPH. We measured CSF A $\beta$ oligomer levels before and after CSF shunting in patients with iNPH.

\section{Methods}

We evaluated two iNPH cohorts: an analysis cohort (cohort-1) with 52 patients and a validation cohort (cohort-2) with 13 patients. For comparison cohorts, we recruited 27 neurologically normal controls (NCs), 16 patients with $A D, 15$ patients with Parkinson's disease (PD), and 14 patients with progressive supranuclear palsy (PSP). We measured CSF A $\beta$ oligomer levels and assessed participants' neurological statuses. We then compared the iNPH cohorts' pre-shunting measurements with the comparison groups' measurements and compared cohort-1's measurements recorded before and after CSF shunting.

\section{Results}

iNPH cohort-1 had higher CSF Aß oligomer levels than the NC, PD, and PSP cohorts. This result was validated with data from iNPH cohort-2. CSF A $\beta$ oligomer levels differentiated iNPH cohort-1 from the PD and PSP groups, with an area under receiver operating characteristic curve of 0.94 . A $\beta$ oligomer levels in iNPH cohort-1 decreased after CSF shunting. However, there was no correlation between $A \beta$ oligomer levels and cognitive functions in iNPH cohort-1.

\section{Conclusion}

The $A \beta$ oligomer accumulates in patients with iNPH patients but can be eliminated with CSF shunting, suggesting that CSF stagnation causes $A \beta$ oligomer accumulation in $\mathrm{iNPH}$.

\section{Introduction}

Idiopathic normal pressure hydrocephalus (iNPH) is a neurodegenerative disease involving gait disturbances, cognitive impairment, and urinary incontinence that was first described by Hakim et al. in 1965.(1) iNPH is a treatable neurodegenerative condition, and $80 \%$ of patients normally improve with cerebrospinal fluid (CSF) shunt surgery.(2) However, its pathophysiology remains unclear. 
According to the recently articulated "oligomer hypothesis", the amyloid-beta (A $\beta$ ) oligomer contributes to cognitive impairments in patients with Alzheimer's disease (AD).(3) The A $\beta$ peptide has a strong tendency to aggregate and the oligomer is considered more toxic than the monomer.(4) It has been reported that a nonamer (called $A \beta^{\star 52}$ ) has strong neurotoxicity,(5) and CSF concentrations of the $A \beta$ oligomer, which usually has 10-20 monomers, are higher in patients with $A D$ than in healthy controls and are correlated with cognitive impairment severities.(6)

We have previously published reports regarding the CSF concentrations of the $A \beta_{42}$ toxic conformer, which usually contains 2-3 monomers, in patients with iNPH. In patients with iNPH the toxic conformer ratio (i.e. the ratio of toxic conformer levels to total $A \beta_{42}$ levels) is higher than in cognitively normal individuals and lower than in patients with AD. Patients with iNPH who have decreased toxic conformer ratios following CSF shunting display better outcomes than patients without such decreases.(7) Impairment of CSF absorption followed by CSF accumulation, impaired CSF secretion, and a brief period of CSF stagnation may play a key role in iNPH pathophysiology,(8) as it may disrupt clearance of waste molecules, such as $A \beta$ peptides, and lead to cognitive impairment.

On the basis of the aforementioned findings, we hypothesised that CSF stagnation in patients with iNPH may result in the accumulation of the $A \beta$ oligomer and that CSF shunting can restore normal clearance of CSF waste products and thus avert cognitive disturbances. To the best of our knowledge, the CSF levels of $A \beta$ oligomers in patients with iNPH have not been systematically investigated. We therefore aimed to measure the CSF A $A$ oligomer concentrations of patients with iNPH and compare them with those of neurologically normal controls and those of patients with other neurodegenerative diseases. We also aimed to determine whether CSF A $\beta$ oligomer concentrations change following CSF shunting and to test for correlations between these biomarkers and cognitive functions.

\section{Materials And Methods}

\section{Study populations}

Our evaluations included two iNPH cohorts: an analysis cohort and a validation cohort. We diagnosed patients with iNPH according to the Japanese iNPH guidelines.(9) We enrolled 173 consecutive patients with iNPH who had undergone lumbo-peritoneal shunting at the Department of Neurosurgery of Juntendo University (Tokyo, Japan) between April 2011 and December 2017. The following patients were excluded from the study: 32 patients aged $<70$ years or $>85$ years, 9 patients without at least 2 years of follow-up after surgery, 9 patients for whom no CSF had been obtained preoperatively or who had a previous tap test history at another institute, and 71 patients who had not been studied with [ ${ }^{123}$ I]-ioflupane singlephoton emission computed tomography (i.e. dopamine transporter scintigraphy [DaTSCAN]). Ultimately, we included 52 patients in the analysis cohort (iNPH cohort-1). For the validation cohort (iNPH cohort-2), we used data from 13 patients who were diagnosed through the neurology department of the National Hospital Organization Takasaki General Medical Centre (Takasaki, Japan) according to the same criteria used for iNPH cohort-1. 
Because patients with iNPH may have additional comorbid neurodegenerative conditions, we subclassified the iNPH cohort-1 patients according to CSF concentrations of phosphorylated tau (pTau) and DaTSCAN findings. On the basis of our previous findings that CSF pTau levels $\geq 30 \mathrm{pg} / \mathrm{mL}$ are associated with worsened cognitive function outcomes 3 years after shunting in patients with iNPH,(10) we assumed patients with pTau levels $>30 \mathrm{pg} / \mathrm{mL}$ had $A D$ comorbidity.

We used the specific binding ratios (SBRs) for [ ${ }^{123}$ I]-ioflupane determined with DaTSCAN to detect Parkinson's spectrum (PS) comorbidities. Given that SBRs vary depending on clinical settings, it is difficult to directly compare SBRs measured at different institutions. However, a previous report from our institution indicated that a cut-off SBR value of 2.83 is a useful diagnostic threshold for diffuse Lewy body disease.(11) We therefore, assumed that patients with SBR values $<3$ had PS comorbidity. The patients in iNPH cohort-1 were thus subclassified into four subgroups: 15 patients in an "iNPH without comorbidities" subgroup, 18 patients in an "iNPH with AD comorbidity" subgroup, 14 patients in an "iNPH with PS comorbidity" subgroup, and 5 patients in an "iNPH with AD/PS comorbidity" subgroup (Fig. 1).

To evaluate the $A \beta$ oligomer levels in patients with iNPH, we also enrolled neurologically normal controls (NCs) and patients with other types of neurodegenerative disease (i.e. AD, Parkinson's disease [PD], and progressive supranuclear palsy [PSP]) into comparison cohorts. Sixteen patients with AD were diagnosed by a neurologist according to criteria for probable AD published by the National Institute of Neurological and Disorders and Stroke and the Alzheimer's Association.(12) Fifteen patients with PD were diagnosed by a neurologist according to the Movement Disorder Society Clinical Diagnostic Criteria for PD.(13) Fourteen patients with PSP were clinically diagnosed by a neurologist according to the Movement Disorder Society criteria.(14) Twenty-seven NCs who were $>60$ years of age and had Mini-Mental State Examination (MMSE) scores of 26 points or higher were enrolled. All NCs had previously undergone lumbar puncture procedures for various reasons, such as investigations into headache aetiologies, preoperative insertion of a lumbar drainage catheter for aortic surgery, undergoing planned myelography for degenerative lumbar spine disease, or undergoing planned surgery with spinal anaesthesia.

\section{Study design}

The iNPH cohort-1 patients were followed for at least 2 years after CSF shunting. CSF samples were obtained preoperatively and 1 year after shunt surgery, as described below. Patients were assessed with the MMSE, the Frontal Assessment Battery (FAB), and the modified Rankin Scale (mRS) by a neuropsychologist and with the Japanese iNPH Grading Scale (iNPHGS) by a neurosurgeon before surgery and 1 and 2 years after surgery. The patients with iNPH were assessed for radiographical features such as the presence or absence of disproportionately enlarged subarachnoid space hydrocephalus (DESH) according to clinical management guidelines for iNPH.(9) We also assessed vascular burdens according to a classification system for deep subcortical white matter hyperintensities $(D S W M H)(15)$ and calculated Evans Index values. CSF biomarkers (i.e. pTau, $A \beta_{38}, A \beta_{42}$, the toxic $A \beta_{42}$ conformer, and $A \beta$ oligomers) were quantified with an enzyme-linked immunosorbent assay (ELISA) kit as described below. 
First, we compared iNPH cohort-1 with the comparison cohorts in terms of baseline characteristics and CSF biomarker levels (Study 1). Second, we validated the results of those analyses with data from iNPH cohort-2 (Study 2). Third, we performed comparisons of the following iNPH cohort-1 subgroups: those without comorbidities, with AD comorbidity, and with PS comorbidity. We compared them in terms of baseline characteristics and CSF biomarker levels (Study $3-1$ ), and before and after CSF shunting in each subgroup (Study $3-2$ ). We compared CSF biomarker levels measured before and 1 year after shunt surgery as well as neurological statuses recorded before and at 1-and 2-year timepoints after shunting. Using data from iNPH without comorbidity subgroup, we analysed the correlations between $A \beta$ oligomer levels and MMSE scores before surgery, and between A $\beta$ oligomer levels 1 year after surgery and MMSE scores 2 years after surgery (Fig. 2).

\section{CSF analysis}

CSF samples were obtained via lumbar puncture performed in the L3-L4 or L4-L5 interspace with an 18gauge spinal needle. Lumbo-peritoneal shunting was performed with our previously reported method.(16) We used an adjustable valve in all patients (Medtronic Neurosurgery, Goleta, CA, or Integra Codman, Raynham, MA). For postoperative follow-up, we adjusted the valve pressure and monitored proper shunt function with CSF withdrawal via valve reservoir puncture as previously reported. $(7,16)$ If shunt malfunction was detected, the patient underwent shunt revision immediately.

To remove cells and debris, CSF samples were centrifuged at $1,690 \mathrm{~g}$ for $10 \mathrm{~min}$ at $4{ }^{\circ} \mathrm{C}$ and then aliquoted and stored in polypropylene tubes at $-80^{\circ} \mathrm{C}$. We used ELISA kits to measure the levels of pTau (T1008; Nipro Corporation, Osaka, Japan), $A \beta_{38}$ (code no.: 27717; IBL Japan, Fujioka, Japan), $A \beta_{42}$ (INNOTEST; code no.: 81583; Fujirebio, Tokyo, Japan), the toxic $A \beta_{42}$ conformer (code no.: 27709; IBL Japan), and A $\beta$ oligomers (code no.: 298-80101; FUJIFILM Wako Pure Chemical Corporation, Osaka Japan). For the AB oligomer ELISA kit, BAN50 was used as both the capture antibody and detector antibody. This kit specifically detects $A \beta$ oligomers containing 10-20 monomers and does not detect monomers, dimers, trimers, tetramers, or hexamers.(6) Luminescence measurements were used to detect our target $A \beta$ oligomers given that these represent $<1 \%$ of $A \beta$ oligomers overall. Therefore, we used a $96-$ well microplate reader (SpectraMax L; Molecular Devices Japan, Tokyo, Japan) and its accompanying software (SoftMax Pro 5.4.8).

\section{Statistics}

Data distribution was evaluated graphically using histograms and Q-Q plots. In study 1, 2 and $3-1$, the Kruskal-Wallis $\mathrm{H}$ test was performed for factorial analysis of variance and the Steel-Dwass test for multiple comparisons. Fisher's exact test was conducted to compare proportions. Areas under receiver operating characteristic curves (AUROCs) were calculated as goodness-of-fit descriptors for predictors of iNPH status versus AD or PD and PSP status. In study $3-2$, the Friedman's test followed by Wilcoxon signed-rank test with Holm's correction was conducted to evaluate differences between neurological statuses before and 1 and 2 years after CSF shunting, and the Wilcoxon signed-rank test was conducted 
to evaluate differences between biomarkers measured before and after CSF shunting. Spearman's rank correlation coefficient was used to evaluate correlations. All statistical analyses were performed with statistical software EZR version 1.41 based on $R$ and $R$ commander.(17) Results are shown as means \pm standard deviations for ages and CSF biomarker values and as medians and interquartile ranges for clinical evaluation battery scores. A $p$-value $<0.05$ was considered statistically significant.

\section{Results}

Comparisons of the iNPH cohorts with the NC, AD, PD, and PSP groups (Studies 1 and 2)

Comparisons of the iNPH cohorts and the NC, AD, PD, and PSP groups revealed significant differences in the sex ratios and the proportions of patients with hypertension and dyslipidaemia. The A $\beta$ oligomer levels in iNPH cohort-1 and the AD group were significantly higher than in the NC, PD, and PSP groups (Figure 3). The A $\beta_{42}$ levels in the AD group were significantly lower than those in iNPH cohort-1 and the NCs $(p=0.014,<$ 0.001 significantly). The pTau levels in the AD group were significantly higher than those in any other group (Table 1). An A $\beta$ oligomer cut-off level of $5.65 \mathrm{pM}$ distinguished iNPH cohort-1 members from AD group members with 50.0\% sensitivity, 76.9\% specificity, and an AUROC curve of 0.61. An A $\beta$ oligomer cut-off level of $5.48 \mathrm{pM}$ distinguished iNPH cohort-1 members from PD and PSP group members with $93.1 \%$ sensitivity, $80.8 \%$ specificity, and an AUROC curve of 0.90 (Figure 4).

In the validation analysis, the iNPH cohort- 2 displayed significantly higher levels of $\mathrm{A} \beta$ oligomer than the NC, PD, and PSP subgroups did. This result is consistent with that observed for the iNPH cohort-1.

Table 1. Baseline characteristics of Study 1 and 2 


\begin{tabular}{|c|c|c|c|c|c|c|c|}
\hline & $\begin{array}{l}\text { iNPH } \\
\text { cohort-1 } \\
(\mathrm{n}=52)\end{array}$ & $\begin{array}{l}\text { iNPH } \\
\text { cohort-2 } \\
(\mathrm{n}=13)\end{array}$ & $\begin{array}{l}\mathrm{AD} \\
(\mathrm{n}=16)\end{array}$ & $\begin{array}{l}\mathrm{PD} \\
(\mathrm{n}=15)\end{array}$ & $\begin{array}{l}\text { PSP } \\
(n=14)\end{array}$ & $\begin{array}{l}\text { NC } \\
(n=27)\end{array}$ & $\begin{array}{l}p- \\
\text { value }\end{array}$ \\
\hline $\begin{array}{l}\text { Ige: mean } \pm \\
\text { SD }\end{array}$ & $\begin{array}{l}76.48 \pm \\
4.17 \\
\end{array}$ & $\begin{array}{l}76.54 \pm \\
4.27 \\
\end{array}$ & $75.63 \pm 7.98$ & $70.80 \pm 8.60$ & $71.00 \pm 11.31$ & $77.48 \pm 6.17$ & 0.191 \\
\hline $\begin{array}{l}\text { jex: } \\
\text { Male number } \\
\%)\end{array}$ & $33(63.5)$ & $5(38.4)$ & $6(37.5)$ & $8(53.3)$ & $11(78.6)$ & $8(29.6)$ & $0.012^{*}$ \\
\hline $\begin{array}{l}\text { MMSE score: } \\
\text { Median [25\% } \\
75 \% \text { ] } \\
\end{array}$ & $\begin{array}{l}24[22- \\
27]\end{array}$ & $\begin{array}{l}22[16- \\
26.5]\end{array}$ & $22[18-22.75]^{*}$ & N.A. & N.A. & $\begin{array}{l}29[28- \\
30] * * * \# \# \#\end{array}$ & \\
\hline \multicolumn{8}{|c|}{ Jomorbidities (number (\%)) } \\
\hline Hypertension & $25(48.1)$ & N.A. & $4(25)$ & $6(40)$ & $6(42.9)$ & $3(11.1)$ & $0.013^{*}$ \\
\hline Jiabetes & $9(17.3)$ & N.A. & $3(18.8)$ & $3(20.0)$ & $6(42.9)$ & $1(3.7)$ & 0.050 \\
\hline Jyslipidaemia & $18(34.6)$ & N.A. & $1(6.3)$ & $4(26.7)$ & $2(14.3)$ & $0(0)$ & $0.002^{* *}$ \\
\hline $\begin{array}{l}\text { Zardiac } \\
\text { liseases }\end{array}$ & $5(9.6)$ & N.A. & $1(6.3)$ & $0(0)$ & $2(14.3)$ & $2(7.4)$ & 0.723 \\
\hline Stroke & $6(12)$ & N.A. & $1(6.3)$ & $1(6.7)$ & $3(21.4)$ & $0(0)$ & 0.146 \\
\hline \multicolumn{8}{|c|}{ 3iomarkers (mean \pm SD) } \\
\hline Jau (pg/ml) & $\begin{array}{l}32.1 \pm \\
16.9 \\
\end{array}$ & $\begin{array}{l}28.0 \pm \\
18.3 \\
\end{array}$ & $\begin{array}{l}102.3 \pm 35.5^{* * *} \\
\# \# \#\end{array}$ & $28.2 \pm 10.8$ & $29.1 \pm 12.7$ & $25.8 \pm 8.4$ & \\
\hline $1 \beta_{42}(\mathrm{pg} / \mathrm{ml})$ & $\begin{array}{l}605.1 \pm \\
262.7 \\
\end{array}$ & $\begin{array}{l}427.7 \pm \\
193.4 \\
\end{array}$ & $388.8 \pm 149.9 *$ & $601.0 \pm 266.3$ & $562.5 \pm 301.0$ & $676.0 \pm 193.9^{\wedge}$ & \\
\hline $\begin{array}{l} \pm \beta \text { oligomer } \\
\text { pM) }\end{array}$ & $\begin{array}{l}6.52 \pm \\
1.65\end{array}$ & $\begin{array}{l}6.73 \pm \\
0.92\end{array}$ & $6.01 \pm 1.18$ & $\begin{array}{l}3.30 \pm 0.88^{* * *} \\
\# \# \#\end{array}$ & $\begin{array}{l}4.54 \pm 0.98 * * \\
\# \#\end{array}$ & $\begin{array}{l}3.53 \pm 1.20 * * * \\
\text { \#\#\# }\end{array}$ & \\
\hline
\end{tabular}

Table 1. Differences in the sex ratios and the percentages of patients with comorbidities were evaluated with Fisher's exact test. Kruskal-Wallis H test was performed for factorial analysis of variance in age and the Steel-Dwass test was performed for multiple comparison in MMSE scores and values of biomarkers. Significance in multiple comparison is shown as ${ }^{*} p<0.05,{ }^{* *} p<0.01$, or ${ }^{* * *} p<0.001$ (versus iNPH cohort-1); $\# p<0.05, \# \# p$ $<0.01$, or \#\#\# $p<0.001$ (versus iNPH cohort-2).

\section{Comparisons of iNPH subgroups (Study 3-1)}

In comparisons of the iNPH cohort-1 without comorbidities, iNPH with AD comorbidity, and iNPH with PS comorbidity subgroups, there were no significant differences in terms of 
sex ratios or the proportions of patients with DESH, DSWMH scores of $0-1$, or comorbidities. The iNPH with PS comorbidity subgroup members were significantly younger than iNPH with AD comorbidity subgroup members. The iNPH without comorbidities subgroup members had significantly higher Evans Index values than the iNPH with PS comorbidity subgroup members did. The iNPH without comorbidity subgroup had significantly higher $A \beta$ oligomer levels than the $\mathrm{iNPH}$ with PS comorbidity subgroup and had significantly lower $A \beta_{38}$ levels than the $\mathrm{iNPH}$ with $\mathrm{AD}$ comorbidity subgroup. The iNPH with AD comorbidity subgroup had significantly higher levels of the toxic $A \beta_{42}$ conformer than the iNPH with PS comorbidity subgroup. There were no significant differences between the subgroups in terms of $A \beta_{42}$ levels (Table 2).

Table 2. Baseline characteristics of Study 3-1 


\begin{tabular}{|c|c|c|c|c|c|c|}
\hline & \multirow{2}{*}{$\begin{array}{l}\text { A: iNPH without } \\
\text { comorbidity } \\
(n=15)\end{array}$} & \multirow{2}{*}{$\begin{array}{l}\text { B: iNPH with } \\
\text { AD } \\
(\mathrm{n}=18)\end{array}$} & \multirow{2}{*}{$\begin{array}{l}\text { C: iNPH with PS } \\
(\mathrm{n}=14)\end{array}$} & \multicolumn{3}{|l|}{$p$-value } \\
\hline & & & & A vs $B$ & A vs $C$ & B vs C \\
\hline Ige: mean \pm SD & $75.93 \pm 4.17$ & $78.11 \pm 3.51$ & $74.00 \pm 2.54$ & 0.263 & 0.501 & $0.006^{* *}$ \\
\hline jex: Male (number (\%)) & $10(66.7)$ & $11(61.1)$ & $8(61.7)$ & \multicolumn{3}{|l|}{0.930} \\
\hline $\begin{array}{l}\text { MMSE score: } \\
\text { median }[25 \%-75 \%]\end{array}$ & $\begin{array}{l}27 \\
{[22-28]}\end{array}$ & $24[22-26]$ & $\begin{array}{l}23.5[21.25- \\
24.75]\end{array}$ & 0.485 & 0.409 & 0.930 \\
\hline Zvans Index: mean \pm SD & $0.363 \pm 0.025$ & $0.341 \pm 0.033$ & $0.336 \pm 0.023$ & 0.054 & $0.027 *$ & 0.993 \\
\hline JESH: number (\%) & $13(86.7)$ & $15(83.3)$ & $12(85.7)$ & \multicolumn{3}{|l|}{1.000} \\
\hline $\begin{array}{l}\text { Nhite matter change: } \\
\text { Number of DSWMH score } 0 \text { - } \\
L(\%)\end{array}$ & $5(33.3)$ & $4(22.2)$ & $6(42.9)$ & \multicolumn{3}{|l|}{0.485} \\
\hline \multicolumn{7}{|l|}{ Jomorbidities (number (\%)) } \\
\hline Hypertension & $7(46.7)$ & $7(38.9)$ & $9(64.3)$ & \multicolumn{3}{|l|}{0.384} \\
\hline Jiabetes & $1(6.7)$ & $4(22.2)$ & $2(14.3)$ & \multicolumn{3}{|l|}{0.457} \\
\hline Jyslipidaemia & $6(40)$ & $7(38.9)$ & $3(21.4)$ & \multicolumn{3}{|l|}{0.543} \\
\hline Jardiac diseases & $2(13.3)$ & $3(16.7)$ & $0(0)$ & \multicolumn{3}{|l|}{0.355} \\
\hline itroke & $1(6.7)$ & $1(5.6)$ & $4(28.6)$ & \multicolumn{3}{|l|}{0.136} \\
\hline \multicolumn{7}{|l|}{ 3iomarkers (mean \pm SD) } \\
\hline JTau (pg/ml) & $22.8 \pm 6.8$ & $43.5 \pm 18.3$ & $22.3 \pm 5.2$ & $<0.001 * * *$ & 0.801 & $<0.001 * * *$ \\
\hline$\pm \beta_{38}(\mathrm{pg} / \mathrm{ml})$ & $2545 \pm 1217$ & $3850 \pm 1556$ & $3372 \pm 1179$ & $0.037 *$ & 0.188 & 0.704 \\
\hline $1 \beta_{42}(\mathrm{pg} / \mathrm{ml})$ & $621.5 \pm 282.6$ & $643.5 \pm 317.2$ & $558.4 \pm 210.7$ & 1.000 & 0.671 & 0.816 \\
\hline $1 \beta_{42}$ toxic conformer $(\mathrm{pg} / \mathrm{ml})$ & $75.53 \pm 35.83$ & $80.67 \pm 32.56$ & $54.79 \pm 12.97$ & 0.868 & 0.377 & $0.040 *$ \\
\hline$\pm \beta$ oligomer $(\mathrm{pM})$ & $7.26 \pm 0.90$ & $6.40 \pm 1.83$ & $5.93 \pm 1.5$ & 0.192 & $0.015^{*}$ & 0.633 \\
\hline
\end{tabular}

Table 2. Differences in age, MMSE scores, Evans Index values, and biomarkers levels were evaluated with the Steel-Dwass test. Differences in sex ratios, DESH prevalence, the percentages of patients with DSWMH scores of $0-1$, and comorbidity prevalence were evaluated with Fisher's exact test. Significance is shown as ${ }^{*} p<0.05,{ }^{* *} p<0.01$, or $* * * p<$ 0.001 . 
In the iNPH without comorbidities subgroup, gait disturbance was significantly improved and maintained for 2 years after CSF shunting. Although there was improvement in cognitive function, as detected by the Friedman's test, there was no statistical significance according to the applied post-hoc Wilcoxon signed-rank test (Additional file 1). In the iNPH with PS comorbidity subgroup, cognitive function was significantly improved and maintained for 2 years after CSF shunting. However, there was no significant improvement in gait disturbance. In the iNPH with AD comorbidity subgroup, gait disturbance was improved and maintained for 2 years after CSF shunting. However, there was no significant improvement in cognitive function.

In the iNPH without comorbidities and iNPH with PS comorbidity subgroups, $A \beta_{38}$ levels were significantly increased and $A \beta$ oligomer levels were significantly decreased relative to their pre-CSF shunting levels. Conversely, in the iNPH with AD comorbidity subgroup, the $A \beta_{38}$ levels were significantly increased, but $A \beta$ oligomer levels were unchanged (Table 3 ). In the iNPH without comorbidities subgroup, two patients showed increases in $A \beta$ oligomer levels (Figure 5). One of those two patients experienced a shunt malfunction during followup and underwent shunt revision. In the iNPH without comorbidity subgroup, there were no significant correlations between $\mathrm{A} \beta$ oligomer levels before surgery and MMSE scores before surgery ( $p=0.949$ ) or between A $\beta$ oligomer levels after surgery and MMSE scores 2 years after surgery $(p=0.469)$.

Table 3. Comparisons of measurements taken before and after CSF shunting in Study 3-2 


\begin{tabular}{|c|c|c|c|c|c|c|c|c|c|c|c|c|}
\hline & \multicolumn{4}{|c|}{$\begin{array}{l}\text { iNPH without comorbidity } \\
(n-15)\end{array}$} & \multicolumn{4}{|c|}{$\begin{array}{l}\text { iNPH with AD } \\
(\mathrm{n}=18)\end{array}$} & \multicolumn{4}{|c|}{$\begin{array}{l}\text { iNPH with PS } \\
(\mathrm{n}=14)\end{array}$} \\
\hline & Before & 1 year & $\begin{array}{l}2 \\
\text { years }\end{array}$ & p-value & Before & 1 year & $\begin{array}{l}2 \\
\text { years }\end{array}$ & p-value & Before & 1 year & $\begin{array}{l}2 \\
\text { years }\end{array}$ & $\mathrm{p}$-value \\
\hline $\begin{array}{l}\text { MMSE score: } \\
\text { Median } \\
{[25 \%-75 \%]}\end{array}$ & $\begin{array}{l}27 \\
{[22-} \\
28]\end{array}$ & $\begin{array}{l}28 \\
{[25.5-} \\
29]\end{array}$ & $\begin{array}{l}28 \\
{[24.5} \\
- \\
28.5]\end{array}$ & $0.036^{*}$ & $\begin{array}{l}23.5 \\
{[22-} \\
26]\end{array}$ & $\begin{array}{l}27 \\
{[23.25} \\
-28]\end{array}$ & $\begin{array}{l}26 \\
{[21.25} \\
-27]\end{array}$ & 0.073 & $\begin{array}{l}23.5 \\
{[22-} \\
24]\end{array}$ & $\begin{array}{l}26.5 \\
{[25} \\
-28.5]^{*}\end{array}$ & $\begin{array}{l}25 \\
{[22-} \\
27]\end{array}$ & $0.018^{*}$ \\
\hline $\begin{array}{l}\text { FAB score: } \\
\text { Median } \\
{[25 \%-75 \%]}\end{array}$ & $\begin{array}{l}12 \\
{[9.5-} \\
13]\end{array}$ & $\begin{array}{l}14 \\
{[12-} \\
16]\end{array}$ & $\begin{array}{l}14 \\
{[13-} \\
16] \\
\end{array}$ & 0.061 & $\begin{array}{l}13 \\
{[9-} \\
15] \\
\end{array}$ & $\begin{array}{l}15 \\
{[14-} \\
15]\end{array}$ & $\begin{array}{l}14 \\
{[13-} \\
14.75]\end{array}$ & 0.052 & $\begin{array}{l}11.5 \\
{[9-} \\
13]\end{array}$ & $\begin{array}{l}13 \\
{[9.25-} \\
14]\end{array}$ & $\begin{array}{l}12 \\
{[9-} \\
13] \\
\end{array}$ & 0.128 \\
\hline $\begin{array}{l}\text { mRS score: } \\
\text { Median } \\
{[25 \%-75 \%]}\end{array}$ & 2 & $\begin{array}{l}1 \\
{[1-} \\
2]^{*} \\
\end{array}$ & $\begin{array}{l}1 \\
{[1-} \\
2]^{*} \\
\end{array}$ & $<0.001^{* * *}$ & $\begin{array}{l}3 \\
{[2-3]}\end{array}$ & $\begin{array}{l}2 \\
{[1.25-} \\
2]\end{array}$ & $\begin{array}{l}2 \\
{[2-3]}\end{array}$ & $0.038^{*}$ & $\begin{array}{l}3 \\
{[3-3]}\end{array}$ & $\begin{array}{l}2 \\
{[1.25-} \\
3]\end{array}$ & $\begin{array}{l}2 \\
{[2-} \\
3] \\
\end{array}$ & $0.017 *$ \\
\hline \multicolumn{13}{|c|}{ iNPHGS score: Median [25\% - 75\%] } \\
\hline Total score & $\begin{array}{l}5 \\
{[4-6]}\end{array}$ & $\begin{array}{l}3 \\
{[1.5-} \\
3.5]^{*}\end{array}$ & $\begin{array}{l}2 \\
{[1-} \\
3]^{*}\end{array}$ & $<0.001^{* * *}$ & $\begin{array}{l}5 \\
{[4-6]}\end{array}$ & $\begin{array}{l}4 \\
{[3-} \\
5]^{*}\end{array}$ & $\begin{array}{l}4 \\
{[3-} \\
5.75]\end{array}$ & $0.002 * *$ & $\begin{array}{l}6 \\
{[4.25-} \\
6.75]\end{array}$ & $\begin{array}{l}3 \\
{[2.25-} \\
5.5]^{*}\end{array}$ & $\begin{array}{l}4 \\
{[3-} \\
5]\end{array}$ & $0.005^{* *}$ \\
\hline $\begin{array}{l}\text { Gait } \\
\text { disturbance }\end{array}$ & $\begin{array}{l}2 \\
{[2-2]}\end{array}$ & $\begin{array}{l}1 \\
{[0.5-} \\
1]^{* *} \\
\end{array}$ & $\begin{array}{l}1 \\
{[0-} \\
1]^{* *}\end{array}$ & $<0.001^{* * *}$ & $\begin{array}{l}2 \\
{[2-} \\
2.75] \\
\end{array}$ & $\begin{array}{l}1 \\
{[1-} \\
2]^{*}\end{array}$ & $\begin{array}{l}1.5 \\
{[1-} \\
2]^{*} \\
\end{array}$ & $0.003^{* *}$ & $\begin{array}{l}2 \\
{[2-3]}\end{array}$ & $\begin{array}{l}1 \\
{[1-2]}\end{array}$ & $\begin{array}{l}2 \\
{[1-} \\
2] \\
\end{array}$ & 0.067 \\
\hline $\begin{array}{l}\text { Cognitive } \\
\text { impairment }\end{array}$ & $\begin{array}{l}1 \\
{[1-2]}\end{array}$ & $\begin{array}{l}1 \\
{[0-} \\
1.5] \\
\end{array}$ & $\begin{array}{l}1 \\
{[0-} \\
1] \\
\end{array}$ & 0.062 & $\begin{array}{l}2 \\
{[1-2]}\end{array}$ & $\begin{array}{l}1 \\
{[1-2]}\end{array}$ & $\begin{array}{l}1 \\
{[1-} \\
2]\end{array}$ & 0.469 & $\begin{array}{l}2 \\
{[1-2]}\end{array}$ & $\begin{array}{l}1 \\
{[0.25-} \\
1]^{* *} \\
\end{array}$ & $\begin{array}{l}1 \\
{[1-} \\
1]^{*} \\
\end{array}$ & $<0.001^{* * *}$ \\
\hline $\begin{array}{l}\text { Urinary } \\
\text { incontinence }\end{array}$ & $\begin{array}{l}2 \\
{[1-} \\
2.5]\end{array}$ & $\begin{array}{l}1 \\
{[0-2]}\end{array}$ & $\begin{array}{l}1 \\
{[0-1]}\end{array}$ & $0.013^{*}$ & $\begin{array}{l}2 \\
{[1-2]}\end{array}$ & $\begin{array}{l}1 \\
{[1-1]}\end{array}$ & $\begin{array}{l}1 \\
{[1-} \\
1.75] \\
\end{array}$ & $0.007^{* *}$ & $\begin{array}{l}2 \\
{[1-2]}\end{array}$ & $\begin{array}{l}1 \\
{[1-} \\
1.75]\end{array}$ & $\begin{array}{l}1 \\
{[1-} \\
1.75] \\
\end{array}$ & $0.011^{*}$ \\
\hline \multicolumn{13}{|c|}{ Biomarker: Mean \pm SD } \\
\hline $\mathrm{A} \beta_{38}(\mathrm{pg} / \mathrm{ml})$ & $\begin{array}{l}2545 \pm \\
1217\end{array}$ & $\begin{array}{l}4238 \pm \\
1349\end{array}$ & N.A. & $<0.001^{* * *}$ & $\begin{array}{l}3851 \pm \\
1556\end{array}$ & $\begin{array}{l}5258 \pm \\
1303\end{array}$ & N.A. & $<0.001^{* * *}$ & $\begin{array}{l}3372 \\
\pm \\
1179 \\
\end{array}$ & $\begin{array}{l}4463 \pm \\
1801\end{array}$ & N.A. & $0.042 *$ \\
\hline $\mathrm{A} \beta_{42}(\mathrm{pg} / \mathrm{ml})$ & $\begin{array}{l}621.5 \\
\pm \\
282.6\end{array}$ & $\begin{array}{l}737.3 \\
\pm \\
294.6\end{array}$ & N.A. & 0.532 & $\begin{array}{l}643.5 \\
\pm \\
317.2\end{array}$ & $\begin{array}{l}748.3 \\
\pm \\
350.1\end{array}$ & N.A. & 0.097 & $\begin{array}{l}558.4 \\
\pm \\
210.7\end{array}$ & $\begin{array}{l}686.1 \pm \\
326.8\end{array}$ & N.A. & 0.091 \\
\hline $\begin{array}{l}\mathrm{A} \beta_{42} \text { toxic } \\
\text { conformer } \\
\text { (pg/ml) }\end{array}$ & $\begin{array}{l}75.53 \\
\pm \\
35.83 \\
\end{array}$ & $\begin{array}{l}87.60 \\
\pm \\
28.81 \\
\end{array}$ & N.A. & 0.083 & $\begin{array}{l}80.67 \\
\pm \\
32.57 \\
\end{array}$ & $\begin{array}{l}94.72 \\
\pm \\
32.04 \\
\end{array}$ & N.A. & $0.026^{*}$ & $\begin{array}{l}54.79 \\
\pm \\
19.97 \\
\end{array}$ & $\begin{array}{l}58.29 \pm \\
24.84\end{array}$ & N.A. & 0.379 \\
\hline $\begin{array}{l}\mathrm{A} \beta \text { oligomer } \\
(\mathrm{pM})\end{array}$ & $\begin{array}{l}7.26 \pm \\
0.90 \\
\end{array}$ & $\begin{array}{l}5.10 \pm \\
2.02 \\
\end{array}$ & N.A. & $0.002^{* *}$ & $\begin{array}{l}6.40 \pm \\
1.83 \\
\end{array}$ & $\begin{array}{l}6.38 \pm \\
2.06 \\
\end{array}$ & N.A. & 0.966 & $\begin{array}{l}5.93 \pm \\
1.50\end{array}$ & $\begin{array}{l}4.80 \pm \\
1.64\end{array}$ & N.A. & $0.036 *$ \\
\hline
\end{tabular}

Table 3. Differences between neurological status recorded before and after shunt surgery were evaluated with the Friedman's test followed by Wilcoxon signed-rank test with Holm's correction. Significance in Friedman's test is shown in the table, and that in post-hoc analysis is shown as only asterisks in the lines of 1 year and 2 years (Before versus 1 year, 
Before versus 2 years significantly). Differences between biomarkers recorded before and after shunt surgery were evaluated with the Wilcoxon signed-rank test, and significance is shown in the table. Significance is shown as ${ }^{*} p<0.05,{ }^{* *} p<0.01$ or ${ }^{* * *} p<0.001$.

\section{Discussion}

\section{$A \beta$ oligomer levels are elevated in patients with iNPH}

In iNPH, CSF stagnation is one of the causes of $A \beta$ deposition followed by cognitive impairment.(8) According to a previous report, CSF concentrations of $A \beta_{42}$ in patients with iNPH are lower than or equal to those observed in NCs.(18) Hypo-metabolism of APP and aggregation of $A \beta$ are considered the causes of these changes in APP-related peptides. $(19,20)$ Accelerated production and deposition of $A \beta$ correlates with the onset of $A D$.(21) $A \beta_{42}$ has a strong tendency to aggregate and forms senile plaques and protofibrils in the brain of patients with AD.(6) However, cognitive impairment severities correlate poorly with $A \beta$ plaque loads but correlate strongly with the levels of soluble $A \beta$ oligomers.(22)

The A $\beta$ oligomer levels in the iNPH cohort-1 were significantly higher than those in the NCs and the patients with PD or PSP. This result was validated with data from iNPH cohort-2. Consistent with the findings of previous research, the $A D$ group had higher $A \beta$ oligomer levels than NCs.(6) This result may imply that $A \beta$ aggregation is a disease-specific phenomenon in iNPH pathophysiology as in $A D$, and that $A \beta$ aggregation may be as strong in the iNPH group as in the AD group. However, we do not think that these results merely reflect the severity in $A \beta$ aggregation. Although the antibody we used in the present study detects mainly 10-20-monomer oligomers,(6) we were unable to evaluate their molecular sizes. Therefore, we cannot exclude the possibility that stronger $A \beta$ aggregation produces larger oligomers and apparently decreases the total oligomer numbers in patients.

Patients with iNPH sometimes show clinical symptoms that are similar to those of other neurodegenerative diseases, which complicates differential diagnosis. According to past reports, ADrelated pathology can be observed in $18-75 \%$ of patients with $\mathrm{iNPH},(23,24)$ and $11-86 \%$ of patients with iNPH exhibit parkinsonism.(25-28) Our results indicate that $A \beta$ oligomers are not good diagnostic biomarkers for differentiating iNPH from AD but are useful for differentiating iNPH from PD and PSP. However, as has been previously reported, AD can be differentiated from iNPH using CSF levels of pTau.

Additionally, $A \beta$ oligomer levels were significantly higher in the iNPH without comorbidities subgroup than in the iNPH with PS comorbidity subgroup. This implies that $A \beta$ oligomer levels are useful for distinguishing PS from other comorbidities in patients with iNPH. Although there were no significant differences in baseline clinical severities between iNPH subgroups, patients in the iNPH with PS comorbidity subgroup might have had PS-related pathophysiological features at baseline. Therefore, the effects of iNPH pathophysiology might have been less severe in the iNPH with PS comorbidity subgroup than in the iNPH without comorbidities subgroup. The fact that the Evans Index values in the iNPH 
without comorbidity subgroup was significantly higher than iNPH with PS comorbidity subgroup and that the patients in iNPH with PS comorbidity subgroup displayed worse outcomes in gait disturbance after CSF shunting are consistent with this speculation. These results also imply that $A \beta$ oligomer levels in patients with iNPH may become elevated as the disease progresses.

\section{$A \beta$ oligomer decreases after CSF shunt placement}

In the iNPH without comorbidities and iNPH with PS comorbidity subgroups, $A \beta_{38}$ levels increased and $A \beta$ oligomer levels decreased after CSF shunting. In the iNPH without comorbidities subgroup, only two patients displayed elevated $A \beta$ oligomer levels, and one of those patients experienced a shunt malfunction and received shunt revision. CSF production is disturbed in $\mathrm{NPH}$, and CSF concentrations of APP-derived peptides increase after shunt surgery. $(29,30)$ It is possible that CSF elimination following shunt surgery improves CSF production and APP metabolism in the periventricular space.(31-33) In the present study, elevated CSF $A \beta_{38}$ levels may reflect an improvement in CSF turnover that resulted in decreased $A \beta$ oligomer levels. On the basis of these results, we can speculate that $A \beta$ oligomer accumulation may be caused by CSF stagnation and may be alleviated by CSF shunting.

Alternatively, in the iNPH with $A D$ comorbidity subgroup, $A \beta$ oligomer levels did not decrease despite the elevated $A \beta_{38}$ levels. This result may imply the difference in $A \beta$ metabolism between iNPH and AD. A $\beta$ is derived from the proteolytic cleavage of APP by $\beta$ - and $\gamma$-secretases. It has been reported that $\gamma$-secretase is more strongly activated than $\beta$-secretase in $\mathrm{NPH}$, although $\beta$-secretase is more strongly activated than $Y$-secretase is in AD.(34) From these facts, we hypothesise that amyloid metabolism in AD is different from that in iNPH, which may be poorly affected by CSF stagnation.

As in AD, cognitive impairment in iNPH may also be affected by $A \beta$ oligomer levels. However, in the present study, we did not observe any correlation between $A \beta$ oligomer levels and MMSE scores. The lack of such correlation might be due to the fact, that iNPH without comorbidity subgroup had better cognitive function at the baseline. Although there was no significant difference in baseline characteristics, the iNPH without comorbidity subgroup manifested higher scores in MMSE at the baseline. These higher scores might affect the correlation between $A \beta$ oligomer levels and MMSE scores at the baseline. Actually, in the present study, iNPH without comorbidity subgroup did not show any significant improvement in cognitive function. We considered it to be a result of a "ceiling" effect.

\section{Study limitations}

The main limitations of the current study were that we had only two cohorts of patients with iNPH and that the number of recruited participants was low. AD comorbidity in patients with iNPH was defined only by pTau levels in CSF, and no pathological features were evaluated. PS comorbidity in patients with iNPH was defined exclusively by DaTSCAN results, which might not have reflected true PS pathology.(33)

We excluded patients with iNPH who had past ischemic events, and there were no significant differences between the iNPH cohort-1 subgroups in the proportions of patients with DSWMH scores of $0-1$. 
However, we did not do further investigations for vascular pathologies, such as performing cerebral blood flow studies. Vascular lesions may affect biomarker measurements and DaTSCAN scan findings.

Although we hypothesised that amelioration of CSF stagnation may improve A $\beta$ oligomer accumulation, we could not quantify CSF flow or the volume of CSF eliminated through the shunt device. Future research should attempt to evaluate the amount of CSF produced and eliminated to test this hypothesis.

\section{Conclusions}

We have successfully quantified CSF A $\beta$ oligomer levels in patients with iNPH for the first time. A oligomer levels were higher in the patients with $\mathrm{NPH}$ who had CSF stagnation than in the comparison groups. $A \beta$ oligomer levels in patients with iNPH decreased after CSF shunting.

\section{Availability Of Data And Materials}

The datasets used and/or analysed during the current study are available from the corresponding author on a reasonable request.

\section{Abbreviations}

$A \beta$ : amyloid-beta

AD: Alzheimer's disease

AUC: area under the curve

CSF: cerebrospinal fluid

DaTSCAN: dopamine transporter scintigraphy

DESH: disproportionately enlarged subarachnoid space hydrocephalus

DSWMH: deep subcortical white matter high intensity

FAB: Frontal Assessment Battery

iNPH: idiopathic normal pressure hydrocephalus

iNPHGS: iNPH Grading Scale

LPS: lumbo-peritoneal shunt

MMSE: Mini-Mental State Examination

mRS: modified Rankin Scale 
NC: normal control

PD: Parkinson's disease

PS: Parkinson's spectrum

PSP: progressive supranuclear palsy

p-Tau: phosphorylated tau

ROC: receiver operating characteristic

SBR: specific binding ratio

\section{References}

1. Hakim S, Adams RD. The special clinical problem of symptomatic hydrocephalus with normal cerebrospinal fluid pressure. Observations on cerebrospinal fluid hydrodynamics. J Neurol Sci. 1965;2(4):307-27.

2. Hashimoto M, Ishikawa M, Mori E, Kuwana N. Study of Ioni. Diagnosis of idiopathic normal pressure hydrocephalus is supported by MRI-based scheme: a prospective cohort study. Cerebrospinal Fluid Res. 2010;7:18.

3. Selkoe DJ. Alzheimer's disease is a synaptic failure. Science. 2002;298:789-91.

4. Hardy J, Selkoe DJ. The amyloid hypothesis of Alzheimer's disease: progress and problems on the road to therapeutics. Science. 2002;297(5580):353-6.

5. Lesné SE, Sherman MA, Grant M, Kuskowski M, Schneider JA, Bennett DA, et al. Brain amyloid- $\beta$ oligomers in ageing and Alzheimer's disease. Brain. 2013;136(5):1383-98.

6. Fukumoto H, Tokuda T, Kasai T, Ishigami N, Hidaka H, Kondo M, et al. High-molecular-weight betaamyloid oligomers are elevated in cerebrospinal fluid of Alzheimer patients. FASEB J. 2010;24(8):2716-26.

7. Akiba C, Nakajima M, Miyajima M, Ogino I, Motoi Y, Kawamura K, et al. Change of Amyloid- $\beta$ 1-42 Toxic Conformer Ratio After Cerebrospinal Fluid Diversion Predicts Long-Term Cognitive Outcome in Patients with Idiopathic Normal Pressure Hydrocephalus. J Alzheimers Dis. 2018;63(3):989-1002.

8. Silverberg GD, Mayo M, Saul T, Rubenstein E, McGuire D. Alzheimer's disease, normal-pressure hydrocephalus, and senescent changes in CSF circulatory physiology: a hypothesis. Lancet Neurol. 2003;2(8):506-11.

9. Mori E, Ishikawa M, Kato T, Kazui H, Miyake H, Miyajima M, et al. Guidelines for management of idiopathic normal pressure hydrocephalus: second edition. Neurol Med Chir (Tokyo). 2012;52(11):775-809.

10. Nakajima M, Miyajima M, Ogino I, Akiba C, Kawamura K, Kamohara C, et al. Preoperative Phosphorylated Tau Concentration in the Cerebrospinal Fluid Can Predict Cognitive Function Three 
Years after Shunt Surgery in Patients with Idiopathic Normal Pressure Hydrocephalus. J Alzheimers Dis. 2018;66(1):319-31.

11. Kamagata K, Nakatsuka T, Sakakibara R, Tsuyusaki Y, Takamura T, Sato K, et al. Diagnostic imaging of dementia with Lewy bodies by susceptibility-weighted imaging of nigrosomes versus striatal dopamine transporter single-photon emission computed tomography: a retrospective observational study. Neuroradiology. 2017;59(1):89-98.

12. McKhann G, Drachman D, Folstein M, Katzman R, Price D, Stadlan EM. Clinical diagnosis of Alzheimer's disease: report of the NINCDS-ADRDA Work Group under the auspices of Department of Health and Human Services Task Force on Alzheimer's Disease. Neurology. 1984;34(7):939-44.

13. Postuma RB, Berg D, Stern M, Poewe W, Olanow CW, Oertel W, et al. MDS clinical diagnostic criteria for Parkinson's disease. Mov Disord. 2015;30(12):1591-601.

14. Hoglinger GU, Respondek G, Stamelou M, Kurz C, Josephs KA, Lang AE, et al. Clinical diagnosis of progressive supranuclear palsy: The movement disorder society criteria. Mov Disord. 2017;32(6):853-64.

15. Shinohara Y, Tohgi H, Hirai S, Terashi A, Fukuuchi Y, Yamaguchi T, et al. Effect of the Ca antagonist nilvadipine on stroke occurrence or recurrence and extension of asymptomatic cerebral infarction in hypertensive patients with or without history of stroke (PICA Study). 1. Design and results at enrollment. Cerebrovasc Dis. 2007;24(2-3):202-9.

16. Nakajima M, Miyajima M, Ogino I, Sugano H, Akiba C, Domon N, et al. Use of external lumbar cerebrospinal fluid drainage and lumboperitoneal shunts with Strata NSC valves in idiopathic normal pressure hydrocephalus: a single-center experience. World Neurosurg. 2015;83(3):387-93.

17. Kanda Y. Investigation of the freely available easy-to-use software 'EZR' for medical statistics. Bone Marrow Transplant. 2013;48(3):452-8.

18. Santangelo R, Cecchetti G, Bernasconi MP, Cardamone R, Barbieri A, Pinto P, et al. Cerebrospinal Fluid Amyloid-beta 42, Total Tau and Phosphorylated Tau are Low in Patients with Normal Pressure Hydrocephalus: Analogies and Differences with Alzheimer's Disease. J Alzheimers Dis. 2017;60(1):183-200.

19. Jeppsson A, Wikkelso C, Blennow K, Zetterberg H, Constantinescu R, Remes AM, et al. CSF biomarkers distinguish idiopathic normal pressure hydrocephalus from its mimics. J Neurol Neurosurg Psychiatry. 2019;90(10):1117-23.

20. Graff-Radford NR. Alzheimer CSF biomarkers may be misleading in normal-pressure hydrocephalus. Neurology. 2014;83(17):1573-5.

21. Jack CR, Knopman DS, Jagust WJ, Petersen RC, Weiner MW, Aisen PS, et al. Tracking pathophysiological processes in Alzheimer's disease: an updated hypothetical model of dynamic biomarkers. Lancet Neurol. 2013;12(2):207-16.

22. Klein WL, Krafft GA, Finch CE. Targeting small Abeta oligomers: the solution to an Alzheimer's disease conundrum? Trends Neurosci. 2001;24(4):219-24. 
23. Cabral D, Beach TG, Vedders L, Sue LI, Jacobson S, Myers K, et al. Frequency of Alzheimer's disease pathology at autopsy in patients with clinical normal pressure hydrocephalus. Alzheimers Dement. 2011;7(5):509-13.

24. Leinonen V, Koivisto AM, Savolainen S, Rummukainen J, Sutela A, Vanninen R, et al. Post-mortem findings in 10 patients with presumed normal-pressure hydrocephalus and review of the literature. Neuropathol Appl Neurobiol. 2012;38(1):72-86.

25. Molde K, Soderstrom L, Laurell K. Parkinsonian symptoms in normal pressure hydrocephalus: a population-based study. J Neurol. 2017;264(10):2141-8.

26. Cucca A, Biagioni MC, Sharma K, Golomb J, Gilbert RM, Di Rocco A, et al. Comorbid Normal Pressure Hydrocephalus with Parkinsonism: A Clinical Challenge and Call for Awareness. Case Rep Neurol Med. 2018;2018:2513474.

27. Akiguchi I, Ishii M, Watanabe $Y$, Watanabe T, Kawasaki T, Yagi H, et al. Shunt-responsive parkinsonism and reversible white matter lesions in patients with idiopathic NPH. J Neurol. 2008;255(9):1392-9.

28. Allali G, Garibotto V, Assal F. Parkinsonism Differentiates Idiopathic Normal Pressure Hydrocephalus from Its Mimics. J Alzheimers Dis. 2016;54(1):123-7.

29. Johanson CE, Duncan JA 3rd, Klinge PM, Brinker T, Stopa EG, Silverberg GD. Multiplicity of cerebrospinal fluid functions: New challenges in health and disease. Cerebrospinal Fluid Res. 2008;5:10.

30. Jeppsson A, Zetterberg H, Blennow K, Wikkelso C. Idiopathic normal-pressure hydrocephalus Pathophysiology and diagnosis by CSF biomarkers. Neurology. 2013;80(15):1385-92.

31. Silverberg GD, Mayo M, Saul T, Carvalho J, McGuire D. Novel ventriculo-peritoneal shunt in Alzheimer's disease cerebrospinal fluid biomarkers. Expert Rev Neurother. 2004;4(1):97-107.

32. Agren-Wilsson A, Lekman A, Sjoberg W, Rosengren L, Blennow K, Bergenheim AT, et al. CSF biomarkers in the evaluation of idiopathic normal pressure hydrocephalus. Acta Neurol Scand. 2007;116(5):333-9.

33. Allali G, Garibotto V, Mainta IC, Nicastro N, Assal F. Dopaminergic imaging separates normal pressure hydrocephalus from its mimics. J Neurol. 2018;265(10):2434-41.

34. Laitera T, Sarajarvi T, Haapasalo A, Puli L, Kauppinen T, Makinen P, et al. Increased gamma-secretase activity in idiopathic normal pressure hydrocephalus patients with beta-amyloid pathology. PLoS One. 2014;9(4):e93717.

\section{Declarations}

\section{ACKNOWLEDGEMENT}

We acknowledge the Laboratory of Proteomics and Biomolecular Science, the Laboratory of Molecular and Biochemical Research, the Research Support Centre, and the Juntendo University Graduate School of Medicine for technical assistance. 


\section{FUNDING}

This work was supported in part by Grants-in-Aid for Scientific Research [grant numbers 16KK0187, 17K10908, 18H02916, 20K09398] from the Japan Society for the Promotion of Science.

\section{AUTHOR INFORMATION}

\section{Affiliations}

Department of Neurosurgery, Juntendo University, Hongo Bunkyo-ku, Tokyo 113-8421, Japan

Kaito Kawamura, Madoka Nakajima, Ikuko Ogino, Hanbing Xu, Chihiro Kamohara, Kostadin Karagiozov, Hajime Arai

Department of Neurosurgery, Juntendo Tokyo Koto Geriatric Medical Centre, Shinsuna Koto-ku, Tokyo 136-0075, Japan

Masakazu Miyajima², Chihiro Akiba

Department of Neurology, Mihara Memorial Hospital, Ota-cho, Isezaki-shi, Gunma 372-0006, Japan Mitsuyasu Kanai

Department of Neurology, Juntendo University, Hongo Bunkyo-ku, Tokyo 113-8421, Japan Yumiko Motoi

Medical Technology Innovation Center, Juntendo University, Hongo Bunkyo-ku, Tokyo 113-8421, Japan Shuko Nojiri

Department of Neurosurgery, Kugayama Hospital, Kita-Karasuyama, Setagaya-ku, Tokyo 157-0061, Japan 


\section{Contributions}

$\mathrm{KK}, \mathrm{MM}, \mathrm{MN}, \mathrm{CA}, \mathrm{SY}$ and $\mathrm{HA}$ are contributed to the study concept, design and collection of CSF samples in iNPH cohort-1 and NCs.. IO and HX carried out CSF assay. $\mathrm{CH}$ carried out neuropsychologic assessment in iNPH cohort-1. SN contributed to the statistical analysis and interpretation. MK contributed to the collection of CSF samples and geographic information of individuals in iNPH cohort-2. YM contributed to the collection of CSF samples and geographic information of individuals in AD, PD and PSP. KK contributed to the correction and ensuring of English in the manuscript. KK carried out all analysis for the study and manuscript was written by all authors. All authors read and approved the final manuscript.

\section{Corresponding Author:}

Corresponding to Kaito Kawamura M.D.

\section{ETHICS DECLARATIONS}

\section{Ethics Approval and Consent to Participate}

This study was approved by the Ethics Committee of Juntendo University (approval no.: 17-022). All study participants or their legal guardians were briefed on the details of the study and provided written informed consent.

\section{Consent for Publication}

Not applicable

\section{Competing Interests}

There are no actual or potential conflicts of interest.

\section{Figures}




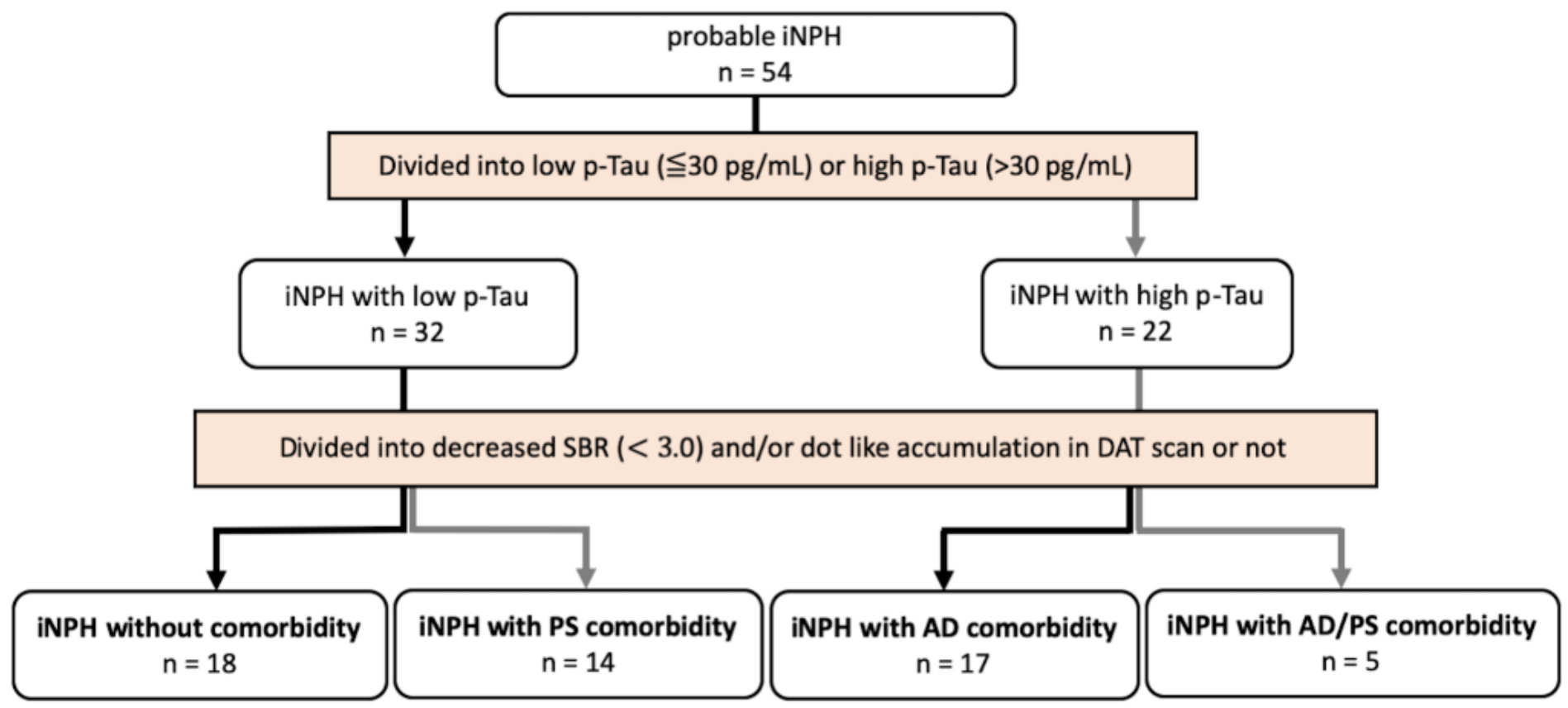

AD: Alzheimer's disease, CSF: cerebrospinal fluid, DaTSCAN: dopamine transporter scintigraphy iNPH: idiopathic normal pressure hydrocephalus, LPS: lumbo-peritoneal shunt, PS: Parkinson's spectrum p-Tau: phosphorylated tau, SBR: specific binding ratio

\section{Figure 1}

Patient enrollment and definitions of analyzed subgroups Patients from iNPH cohort-1 were subclassified according to CSF pTau concentrations and DaTSCAN results. Patients from iNPH cohort-1 were subclassified into an iNPH without comorbidities subgroup, an iNPH with AD comorbidity subgroup, an iNPH with PS comorbidity subgroup, and an iNPH with AD/PS comorbidity subgroup.

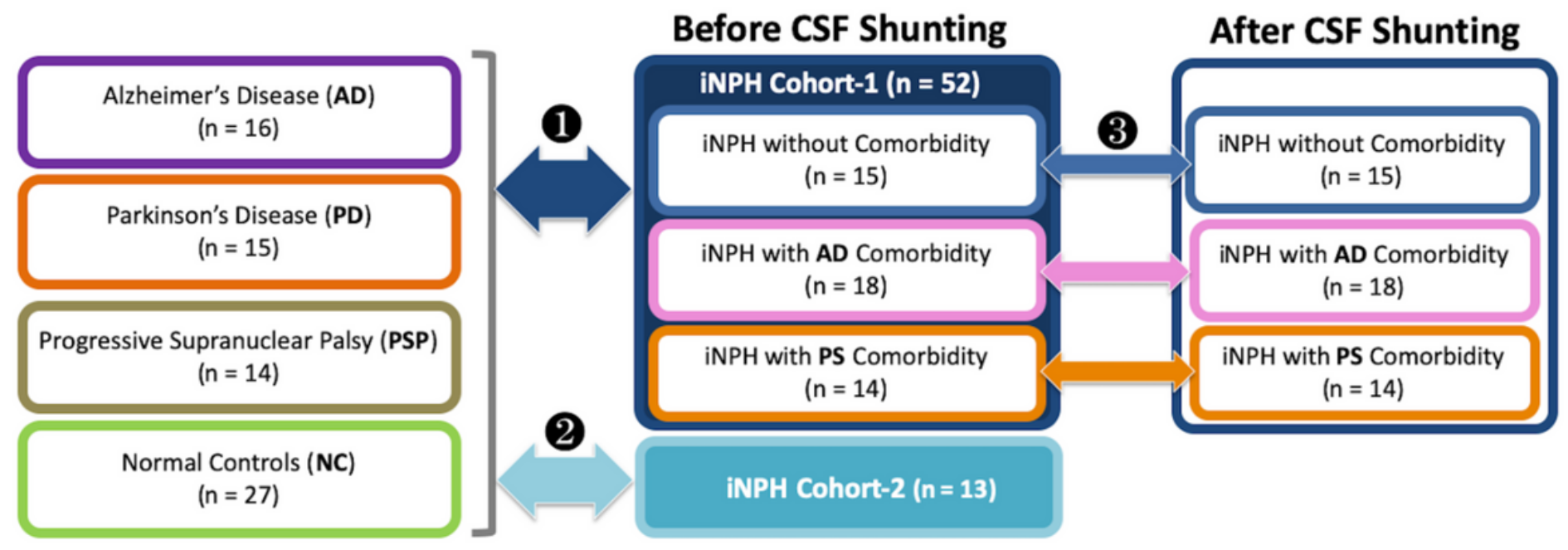

AD: Alzheimer's disease, CSF: cerebrospinal fluid, iNPH: idiopathic normal pressure hydrocephalus, NC: normal control PD: Parkinson's disease, PS: Parkinson's spectrum, PSP: progressive supranuclear palsy

Figure 2 
Study designs We evaluated two iNPH cohorts: the analysis cohort (iNPH cohort-1) with 52 patients and the validation cohort (iNPH cohort-2) with 13 patients. For comparison groups, we also enrolled $27 \mathrm{NCs}$, 16 patients with $A D, 15$ patients with PD, and 14 patients with PSP. In Study 1, we compared iNPH cohort1 with the comparison groups (i.e. the AD, PD, PSP, and NC groups). In Study 2, we compared iNPH cohort2 with the comparison groups to validate the results of Study 1. In Study 3, we compared three iNPH cohort-1 subgroups (i.e. the iNPH without comorbidities, iNPH with AD comorbidity, and iNPH with PS comorbidity subgroups) in terms of baseline characteristics and CSF biomarker levels and in terms of measurements recorded before and after CSF shunting.

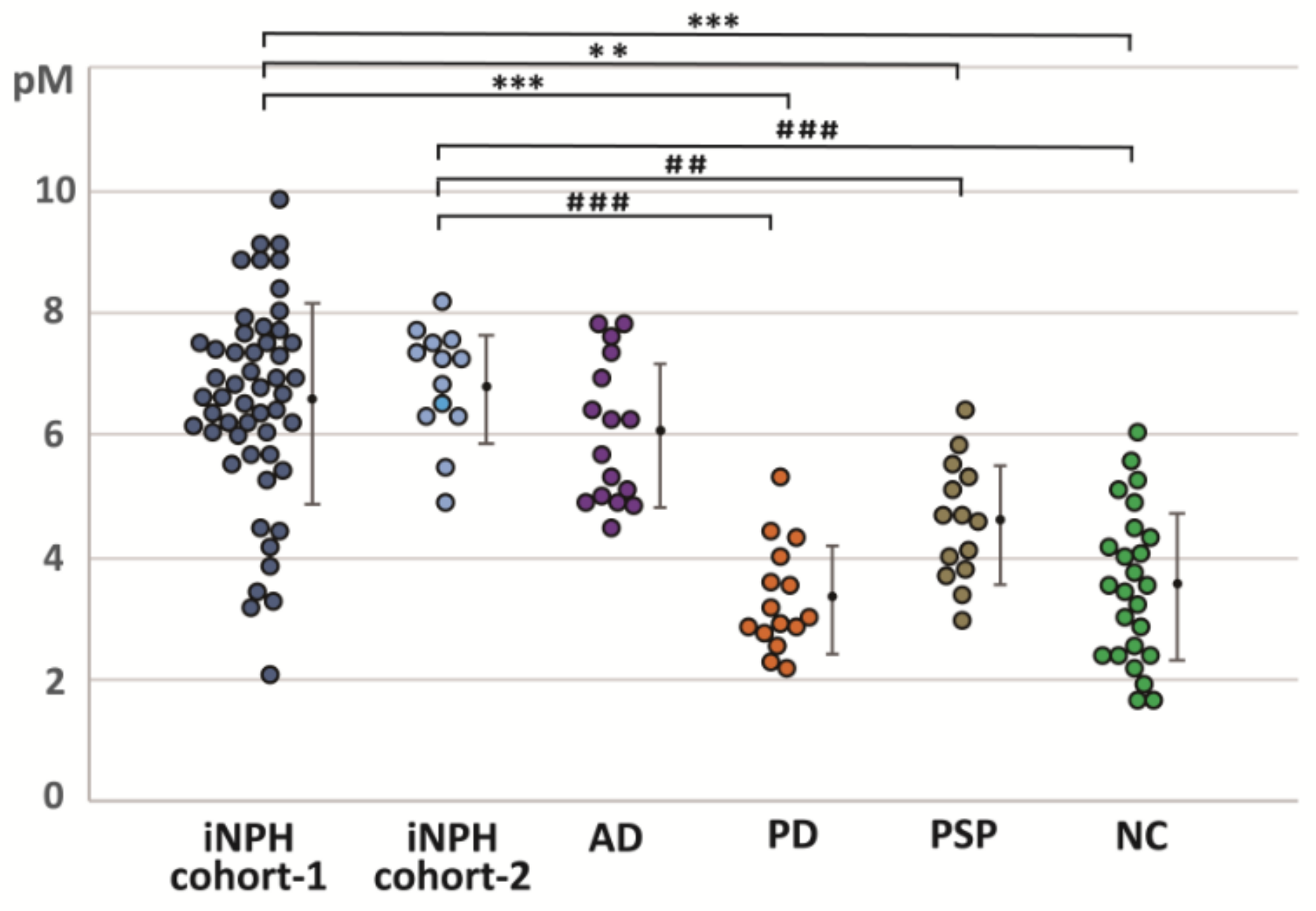

$A \beta$ : amyloid-beta, AD: Alzheimer's disease, CSF: cerebrospinal fluid iNPH: idiopathic normal pressure hydrocephalus, NC: normal control, PD: Parkinson 's disease PSP: progressive supranuclear palsy

\section{Figure 3}

$A \beta$ oligomer levels in each group $A \beta$ oligomer levels for each individual group are represented in a scatter plot. The vertical axis shows the CSF concentrations of $A \beta$ oligomers. Significance testing was done with the Steel-Dwass test and is indicated as ${ }^{\star \star} p<0.01$ or ${ }^{\star \star \star} p<0.001$ (Versus iNPH cohort-1) and \#\#p 0.01 or \#\#\#p<0.001 (Versus iNPH cohort-2). 
A iNPH cohort-1 vs. AD

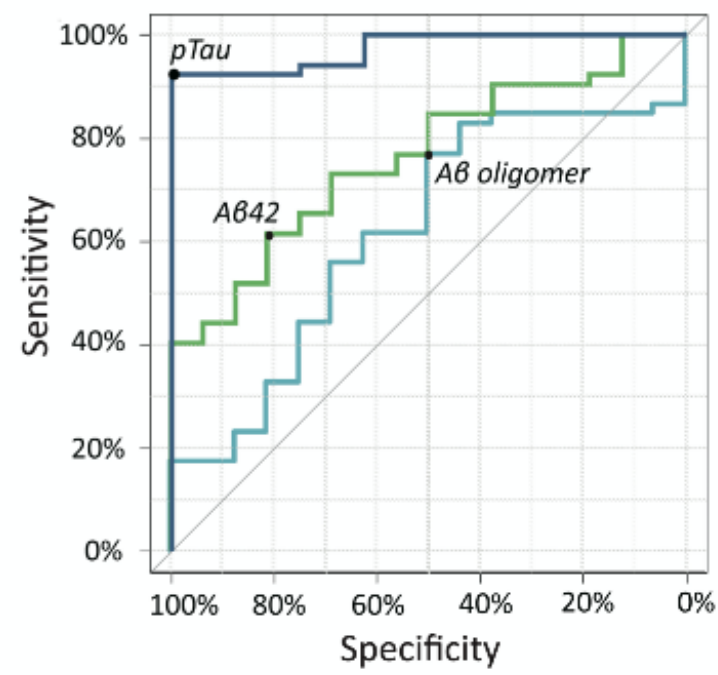

$B$ iNPH cohort-1 vs. PD/PSP

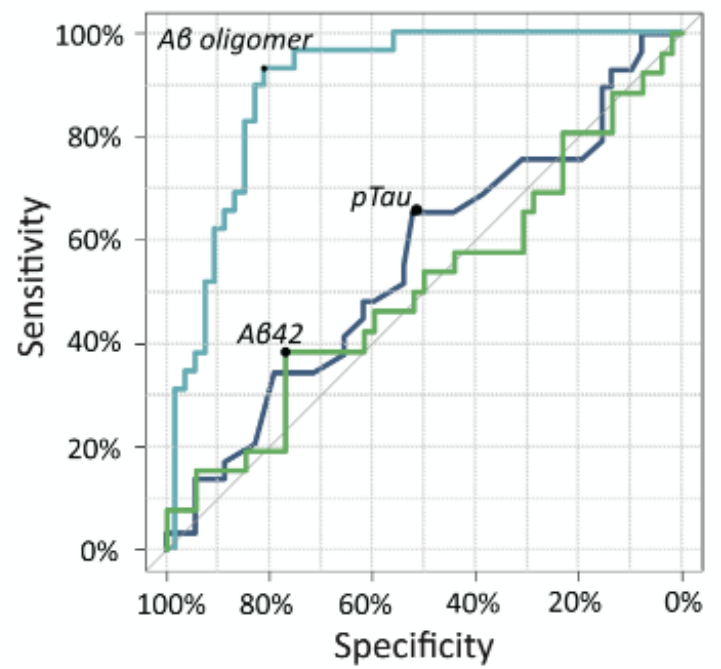

\begin{tabular}{|c|c|c|c|c|c|}
\hline & AUC & $95 \% \mathrm{Cl}$ & Cut-off & Sensitivity & Specificity \\
\hline A6 oligomer & 0.614 & $0.459-0.770$ & $5.656 \mathrm{pM}$ & $50.0 \%$ & $76.9 \%$ \\
\hline A642 & 0.762 & $0.641-0.883$ & $516 \mathrm{pg} / \mathrm{ml}$ & $61.5 \%$ & $81.2 \%$ \\
\hline pTau & 0.974 & $0.943-1.00$ & $54 \mathrm{pg} / \mathrm{ml}$ & $92.3 \%$ & $100 \%$ \\
\hline
\end{tabular}

\begin{tabular}{|c|c|c|c|c|c|}
\hline & AUC & $95 \% \mathrm{Cl}$ & Cut-off & Sensitivity & Specificity \\
\hline A6 oligomer & 0.898 & $0.830-0.966$ & $5.478 \mathrm{pM}$ & $93.1 \%$ & $80.8 \%$ \\
\hline A642 & 0.510 & $0.366-0.653$ & $366 \mathrm{pg} / \mathrm{ml}$ & $38.5 \%$ & $76.9 \%$ \\
\hline pTau & 0.556 & $0.422-0.689$ & $29 \mathrm{pg} / \mathrm{ml}$ & $65.5 \%$ & $51.9 \%$ \\
\hline
\end{tabular}

$\mathrm{A} \beta$ : amyloid-beta, AUC: area under the curve, iNPH: idiopathic normal pressure hydrocephalus p-Tau: phosphorylated tau, ROC: receiver operating characteristic

\section{Figure 4}

ROC analysis of biomarkers ROC analysis of $A \beta$ oligomer levels (blue), $A \beta 42$ levels (green), and pTau levels (navy) as tools for differentiating groups. A, A $\beta$ oligomer levels differentiated members of iNPH cohort-1 from members of the AD groups with an AUC value of 0.614 . $B, A \beta$ oligomer levels differentiated members of the iNPH without comorbidities subgroup from members of the PD and PSP groups with an AUC value of 0.898 .

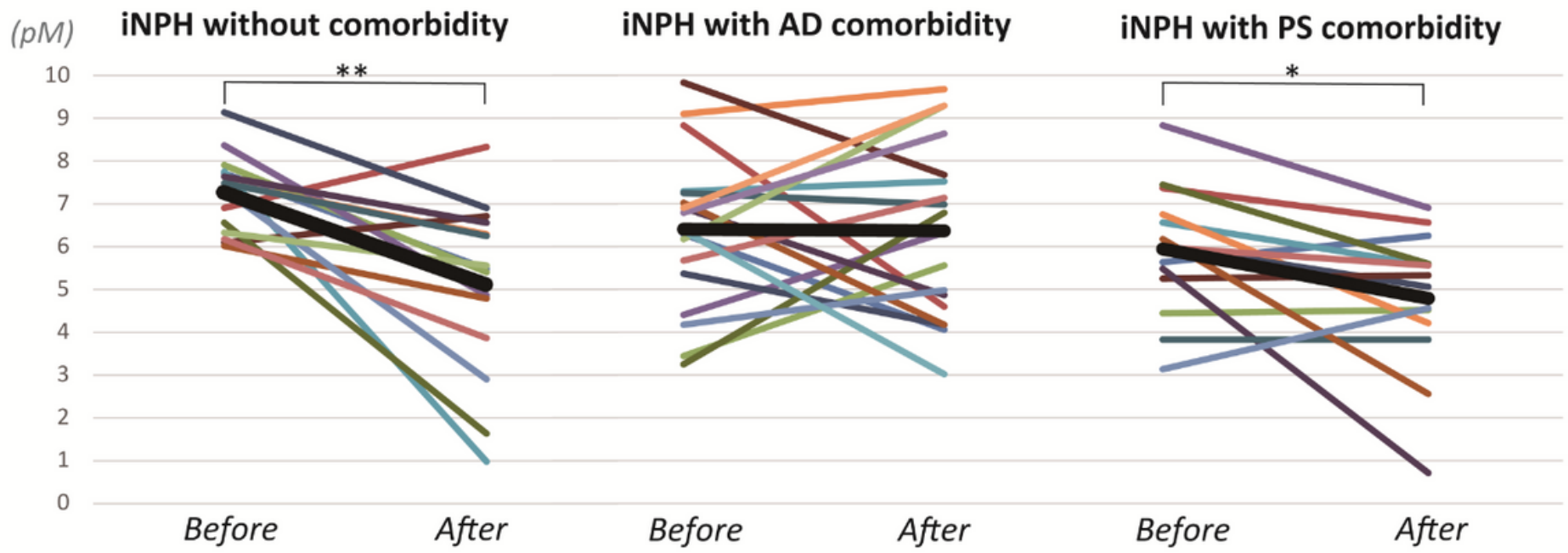

$A \beta$ : amyloid-beta, AD: Alzheimer's disease, CSF: cerebrospinal fluid iNPH: idiopathic normal pressure hydrocephalus, PS: Parkinson's spectrum 


\section{Figure 5}

$A \beta$ oligomer levels before and after shunt surgery $A \beta$ oligomer levels for each study participant are shown in the line graphs and average levels of $A \beta$ oligomer are shown as black lines. The $A \beta$ oligomer level changes after shunt surgery in each subgroup were evaluated with the Wilcoxon signed-rank test. Significant decreases in A $A$ oligomer levels 1 year after CSF shunting were observed in the iNPH without comorbidities subgroup $(p=0.002)$ and the iNPH with PS comorbidity subgroup $(p=0.036$, respectively).

\section{Supplementary Files}

This is a list of supplementary files associated with this preprint. Click to download.

- AdditionalTableJARTFinal.docx 